\title{
Quadrillion $F$-Theory Compactifications with the Exact Chiral Spectrum of the Standard Model
}

\author{
Mirjam Cvetič, ${ }^{1,2}$ James Halverson, ${ }^{3}$ Ling Lin®, ${ }^{1}$ Muyang Liu, ${ }^{1}$ and Jiahua Tian ${ }^{3}$ \\ ${ }^{1}$ Department of Physics and Astronomy, University of Pennsylvania, Philadelphia, Pennsylvania 19104-6396, USA \\ ${ }^{2}$ Center for Applied Mathematics and Theoretical Physics, University of Maribor, SI-2000 Maribor, Slovenia \\ ${ }^{3}$ Department of Physics, Northeastern University, Boston, Massachusetts 02115-5000, USA
}

(Received 27 March 2019; revised manuscript received 19 June 2019; published 6 September 2019)

\begin{abstract}
We present $\mathcal{O}\left(10^{15}\right)$ string compactifications with the exact chiral spectrum of the standard model of particle physics. This ensemble of globally consistent $F$-theory compactifications automatically realizes gauge coupling unification. Utilizing the power of algebraic geometry, all global consistency conditions can be reduced to a single criterion on the base of the underlying elliptically fibered Calabi-Yau fourfolds. For toric bases, this criterion only depends on an associated polytope and is satisfied for at least $\mathcal{O}\left(10^{15}\right)$ bases, each of which defines a distinct compactification.
\end{abstract}

DOI: 10.1103/PhysRevLett.123.101601

Introduction and summary.-As a theory of quantum gravity that naturally gives rise to rich gauge sectors at low energies, string theory is a leading candidate for a unified theory. Achieving unification is an ambitious goal that requires accounting for all aspects of our physical world, which includes not only a rich cosmological history, but also the detailed structure of the standard model of particle physics.

In this Letter, we present an explicit construction that guarantees the existence of $\mathcal{O}\left(10^{15}\right)$ fully consistent string compactifications, which realize the exact chiral particle spectrum of the minimally supersymmetric standard model (MSSM). This construction is performed in the framework of $F$ theory [1], a strongly coupled generalization of type IIB superstring theory. It captures the nonperturbative backreactions of 7-branes onto the compactification space $B_{3}$ in terms of an elliptically fibered Calabi-Yau fourfold $\pi: Y_{4} \rightarrow B_{3}$ over it. Gauge symmetries, charged matter, and Yukawa couplings are then encoded beautifully by $Y_{4}$ 's singularity structures in codimensions one, two, and three, respectively. (We refer the interested reader to [2] and references therein for recent reviews on $F$ theory.)

In the present Letter, we consider a class of elliptically fibered Calabi-Yau fourfolds giving rise to precisely the three-generation MSSM spectrum, provided certain geometric conditions on the base of the fibration are satisfied. We perform a concrete analysis, finding $\mathcal{O}\left(10^{15}\right)$ such bases. All these models come equipped with

Published by the American Physical Society under the terms of the Creative Commons Attribution 4.0 International license. Further distribution of this work must maintain attribution to the author(s) and the published article's title, journal citation, and DOI. Funded by SCOAP ${ }^{3}$. moduli-dependent quark and lepton Yukawa couplings, as well as gauge coupling unification at the compactification scale.

The existence of a very large number of standard model realizations in string theory could perhaps be anticipated within the set of an even larger number of string compactifications (see, e.g., [3]) that form the so-called string landscape. Indeed, though standard model realizations within the landscape could potentially be scarce [4], recent works hint towards an astronomical number of them [5]. Our construction explicitly demonstrates this possibility, increasing the number of concretely known global standard model compactifications in string theory by about 10 orders of magnitude.

There are also explicit constructions of the standard model in other corners of string theory. Some of the early examples of globally consistent intersecting brane models [6] in type II compactifications (see also [7] and references therein) were strongly constrained by global consistency conditions such as tadpole cancellation. In the heterotic string, the typical difficulties of constructions like [8-10] arise from having a stable hidden bundle and the existence of Yukawa couplings. These issues are solved elegantly in $F$ theory through the geometrization of nonperturbative stringy effects: (almost all) global conditions analogous to tadpole cancellation or bundle stability are automatically taken care of by having a compact, elliptic Calabi-Yau fourfold $Y_{4}$, and the presence or absence of Yukawa couplings can be easily read off from codimension three singularities of $Y_{4}$. (In $F$ theory, D3-tadpole cancellation requires extra care and will be a major theme in our constructions.)

Despite these advantages, only a handful [11,12] of $F$-theory compactifications that realize the exact chiral spectrum of the MSSM are currently known, due to 
focusing on a very simple base, $B_{3}=\mathbb{P}^{3}$. This limitation will be avoided in the current Letter by instead studying smooth toric varieties, which provide a much larger class $[13,14]$ of geometries. To take advantage of this large ensemble, we first construct a class of elliptic fibrations (based on the class $\mathbb{P}_{F_{11}}$ in [15]) that can be consistently fibered over all such toric threefolds.

Every such fibration realizes the precise standard model gauge group $[\mathrm{SU}(3) \times \mathrm{SU}(2) \times \mathrm{U}(1)] / \mathbb{Z}_{6}$ as well as its matter representations and Yukawa couplings $[11,15,16]$. Moreover, all models exhibit gauge coupling unification at the compactification scale, compatible with the existence of a complex structure deformation to a geometry realizing the Pati-Salam model with unified gauge coupling $[11,15]$.

Furthermore, for each compatible $B_{3}$ there exists a $G_{4}$ flux that induces three families of chiral fermions. These models have a particularly pleasant feature: all global consistency conditions on the flux (including quantization and $D 3$-tadpole cancellation) can be reduced to a single criterion on the intersection number $\overline{\mathcal{K}}^{3}$ of the anticanonical class $\overline{\mathcal{K}}$ of the base $B_{3}$. For toric threefolds that have a description in terms of a reflexive polytope $\Delta, \overline{\mathcal{K}}^{3}$ depends only on the point configuration of $\Delta$ and not its triangulation. On the other hand, for a single polytope there can be multiple different toric threefolds associated with the different fine-regular-star triangulations (FRSTs) of $\Delta$, the number of which grows exponentially with the number of lattice points in the polytope [13]. Putting together these different components, we find that the number $N_{\mathrm{SM}}^{\text {toric }}$ of globally consistent three-family standard models in our construction is

$$
7.6 \times 10^{13} \lesssim N_{\mathrm{SM}}^{\mathrm{toric}} \lesssim 1.6 \times 10^{16} .
$$

We emphasize that this number is construction dependent; $F$ theory could realize more standard models.

The detailed derivation of this count first requires the construction in Sec. II of a class of elliptic fibrations with a flux inducing three chiral families. All flux consistency conditions reduce to a single criterion on the base $B_{3}$. To count how many $B_{3}$ satisfy this criterion, we discuss the methods to construct FRSTs of 3D polytopes in Sec. III, which ultimately lead us to $\mathcal{O}\left(10^{15}\right)$ possibilities. We close in Sec. IV with some geometric and physical comments, as well as future directions.

Universally consistent fibrations with three families.The class of elliptic fibrations we are interested in is based on an elliptic curve that is a specialized cubic inside $\mathbb{P}^{2}$ with homogeneous coordinates $[u: v: w]$, given by the vanishing of the polynomial

$P:=s_{1} u^{3}+s_{2} u^{2} v+s_{3} u v^{2}+s_{5} u^{2} w+s_{6} u v w+s_{9} v w^{2}$.

By promoting the coefficients $s_{i}$ to rational functions over a Kähler threefold $B_{3}$, one obtains a singular, elliptically fibered fourfold $\pi: Y_{4}^{(s)} \rightarrow B_{3}$. For $Y_{4}^{(s)}$ to be a Calabi-Yau fourfold, the functions $s_{i}$ have to be holomorphic sections of line bundles on $B_{3}$ with first Chern classes $\left[s_{i}\right] \in$ $H^{1,1}\left(B_{3}, \mathbb{Z}\right)$ given by $[11,15]$

$$
\begin{aligned}
& {\left[s_{1}\right]=3 \overline{\mathcal{K}}-\mathcal{S}_{7}-\mathcal{S}_{9}, \quad\left[s_{2}\right]=2 \overline{\mathcal{K}}-\mathcal{S}_{9}, \quad\left[s_{6}\right]=\overline{\mathcal{K}},} \\
& {\left[s_{3}\right]=\overline{\mathcal{K}}+\mathcal{S}_{7}-\mathcal{S}_{9}, \quad\left[s_{5}\right]=2 \overline{\mathcal{K}}-\mathcal{S}_{7}, \quad\left[s_{9}\right]=\mathcal{S}_{9},}
\end{aligned}
$$

where $\overline{\mathcal{K}} \equiv c_{1}\left(B_{3}\right)$ is the anticanonical class of $B_{3}$. The classes $\mathcal{S}_{7,9} \in H^{1,1}\left(B_{3}, \mathbb{Z}\right)$ parametrize different fibrations over the same base, on which $\left\{s_{i}=0\right\}$ define effective divisors.

When all $s_{i}$ are generic (that is, irreducible and $s_{i} \neq s_{j}$ for $i \neq j), F$-theory compactified on $Y_{4}^{(s)}$ has the gauge symmetry $[\mathrm{SU}(3) \times \mathrm{SU}(2) \times \mathrm{U}(1)] / \mathbb{Z}_{6} \quad[15,16]$. The global gauge group structure is reflected in the precise agreement between the geometrically realized matter representations and those of the standard model,
$(\mathbf{3}, \mathbf{2})_{\frac{1}{6}}$,
$(\mathbf{1}, \mathbf{2})_{-\frac{1}{2}}$,
$(\overline{\mathbf{3}}, \mathbf{1})_{-\frac{2}{3}}$,
$(\overline{\mathbf{3}}, \mathbf{1})_{\frac{1}{3}}$,
$(\mathbf{1}, \mathbf{1})_{1}$.

These data can be extracted via the $M-/ F$-theory duality from an explicit resolution $Y_{4}$ of $Y_{4}^{(s)}$, which preserves the Calabi-Yau structure.

A chiral spectrum in $F$ theory requires a nonzero flux $G_{4} \in H^{2,2}\left(Y_{4}\right)$, which must also be specified. For the relevant subspace of so-called primary vertical $G_{4}$ fluxes, there is by now a large arsenal of computational methods $[17,18]$ (see also $[11,19,20]$ ) that allows us to determine base independently the most general flux on $Y_{4}$.

For physical consistency, this $G_{4}$ flux has to satisfy certain conditions. The first condition is a proper quantization $[21,22]$

$$
G_{4}+\frac{1}{2} c_{2}\left(Y_{4}\right) \in H^{2,2}\left(Y_{4}, \mathbb{Z}\right),
$$

where $c_{2}\left(Y_{4}\right)$ is the second Chern class of $Y_{4}$. Heuristically, this condition ensures that the notion of fermions (which requires a flux-dependent spin structure on subspaces of $\left.Y_{4}\right)$ is well defined. Since explicitly verifying this condition for concrete geometries is difficult, we will content ourselves with the usual necessary consistency checks $[11,12,18,19,23]$. The second consistency condition is a D3 tadpole satisfying [24]

$$
n_{D 3}=\frac{\chi\left(Y_{4}\right)}{24}-\frac{1}{2} \int_{Y_{4}} G_{4} \wedge G_{4} \stackrel{!}{\in} \mathbb{Z}_{\geq 0} .
$$

While integrality follows as a consequence of the quantization condition (5), positivity aids in ensuring the stability of the compactification.

We must also impose phenomenological constraints on the flux. A massless electroweak hypercharge $\mathrm{U}(1)_{Y}$ is guaranteed if the $D$-terms vanish $[25,26]$, 


$$
\forall \eta \in H^{1,1}\left(B_{3}\right): \int_{Y_{4}} G_{4} \wedge \sigma \wedge \pi^{*} \eta \stackrel{!}{=} 0 .
$$

Here, $\sigma$ is the $(1,1)$-form Poincare dual to the so-called Shioda-divisor associated with the U(1) [27]. A threefamily chiral standard model requires that [28]

$$
\chi(\mathbf{R})=\int_{\gamma_{\mathbf{R}}} G_{4} \stackrel{!}{=} 3,
$$

for all representations $\mathbf{R}$ in (4). The geometric data $c_{2}\left(Y_{4}\right)$, $\chi\left(Y_{4}\right)$ and the matter surfaces $\gamma_{\mathbf{R}}$ were computed in $[11,15]$. In the Supplemental Material [29], we provide the explicit expression of the generic vertical flux in the resolution $Y_{4}$ presented in [15] and explain in detail how the above conditions can be checked using well-studied topological methods.

We now present our main result: how these consistency conditions can be satisfied for a large ensemble of explicit geometries. For that, we first consider the generic flux configuration on (smooth) fibrations $Y_{4}$ with $\mathcal{S}_{7,9}=\overline{\mathcal{K}}$, which simplifies the expressions for the topological quantities (6)-(8). In fact, one can show that all consistency conditions are reduced to a single criterion on $B_{3}$ from the D3 tadpole

$$
n_{D 3}=12+\frac{5}{8} \overline{\mathcal{K}}^{3}-\frac{45}{2 \overline{\mathcal{K}}^{3}} \stackrel{!}{\in} \mathbb{Z}_{\geq 0},
$$

where $\overline{\mathcal{K}}^{3}$ denotes the triple self-intersection number of the anticanonical class $\overline{\mathcal{K}}$ of the base. This dramatic simplification only requires $\overline{\mathcal{K}}^{3}$ of appropriate value and a base that allows irreducible and distinct $s_{i}$, all of which are sections of the anticanonical class.

In summary, we have constructed a class of elliptically fibered Calabi-Yau fourfolds that gives rise in $F$ theory to the standard model gauge group and matter representations with three chiral generations. The only consistency requirement that guarantees flux quantization and D3-tadpole cancellation is that the base $B_{3}$ of the fibration is a smooth Kähler threefold with nonrigid irreducible anticanonical divisors that satisfy (9). In fact, some basic arithmetic shows that the only values $\overline{\mathcal{K}}^{3}$ can take such that $n_{D 3} \in \mathbb{Z}_{\geq 0}$ are

$$
\overline{\mathcal{K}}^{3} \in\{2,6,10,18,30,90\} .
$$

Counting standard model geometries.-Any smooth threefold $B_{3}$ with nonrigid anticanonical divisors satisfying (10) realizes a globally consistent three-family MSSM in $F$ theory. A subset of such spaces, which can be enumerated combinatorially, is the set of weak Fano toric threefolds encoded by 3D reflexive polytopes $\Delta$. While there are "only" 4319 such polytopes [30], each $\Delta$ can specify inequivalent manifolds $B_{3}$ through different FRSTs of the polytope, whose numbers can be very large [13].
What makes this ensemble particularly attractive for our purpose is the fact that the intersection number $\overline{\mathcal{K}}^{3}$ is determined solely by the polytope $\Delta$ and is completely triangulation independent. Therefore, any $B_{3}$ associated with a FRST of $\Delta$ gives rise to a consistent chiral threegeneration MSSM by our construction, provided that the triangulation-independent constraint on $\overline{\mathcal{K}}^{3}$ is satisfied. In fact, there is a set $S$ with 708 polytopes that satisfy (10). By our construction, we immediately have

$$
N_{\mathrm{SM}}^{\mathrm{toric}}=\sum_{\Delta \in S} N_{\mathrm{FRST}}(\Delta)
$$

where $N_{\text {FRST }}(\Delta)$ is the number of FRSTs of $\Delta$.

Hence, the problem of counting the number of consistent $F$-theory models that admit the chiral MSSM spectrum by our construction reduces to counting FRSTs of reflexive polytopes.

Since $N_{\mathrm{FRST}}(\Delta)$ grows exponentially with the number of lattice points in $\Delta$, the set of consistent threefolds $B_{3}$ is dominated by triangulations of the largest polytope [13], labeled $\Delta_{8}$ in the list of [30]. The FRSTs of this polytope (with $\overline{\mathcal{K}}^{3}=6$ and 39 lattice points) cannot be all constructed explicitly using standard computer programs such as SageMath [31]. To enumerate them, we therefore follow the strategy put forward in [13] to provide bounds on $N_{\text {FRST }}\left(\Delta_{8}\right)$.

The idea is to reduce the complexity by first counting the number of fine-regular triangulations (FRTs) of each facet of a polytope $\Delta$. Since the facets are two-dimensional polytopes, it is possible to use brute force on the combinatorics of FRTs for (almost) all polytopes' facets. (For facets with more than 15 lattice points, using brute force on FRTs also becomes computationally too costly. For these facets, we use different methods outlined in [13] to obtain lower and upper bounds for the number of FRTs.) By virtue of the reflexivity of $\Delta$, any combination of FRTs of all its facets yields fine-star triangulation of $\Delta$.

The drawback of this approach is that the triangulation of $\Delta_{8}$ obtained this way is not guaranteed to be regular. To tackle this issue, we randomly pick $1.3 \times 10^{4}$ samples out of $\mathcal{O}\left(10^{9}\right)$ fine-star triangulations constructed by gluing together FRTs of the facets $\Delta_{8}$. Out of these samples, we find roughly $\frac{2}{3}$ to be also regular triangulations. Combining the factor $\frac{2}{3}$ with the bounds of fine-star triangulations for $\Delta_{8}$ [13], we then obtain $2.6 \times 10^{13} \leq N_{\mathrm{FRST}}\left(\Delta_{8}\right) \leq$ $1.6 \times 10^{16}$.

For the other polytopes in $S$ [i.e., those leading to threefolds satisfying (10)], we can either compute all FRSTs, or we can resort to a similar estimation as with $\Delta_{8}$ if the polytope is too large to use brute force on all FRSTs. We find that these other polytopes sum up to only $\sim 5 \times 10^{13}$ FRSTs, which confirms the dominance of $\Delta_{8}$. In total, we therefore expect the number of consistent 
three-family $F$-theory standard models in our construction over toric threefold bases to be

$$
7.6 \times 10^{13} \lesssim N_{\mathrm{SM}}^{\mathrm{toric}} \lesssim 1.6 \times 10^{16} .
$$

Discussion and outlook.-We have presented a construction that ensures the existence of $\mathcal{O}\left(10^{15}\right)$ explicit globally consistent string compactifications having the exact chiral spectrum of the standard model within the framework of $F$ theory. To our knowledge, this is the largest such ensemble in literature, outnumbering existing results by about 10 orders of magnitude. The models arise by varying the base of one "universal" class of elliptic fibrations introduced in $[11,15]$. We have only focused on the set of toric bases, which already produces around a quadrillion examples. However, we expect that the ensemble of standard models arising from our construction is of orders of magnitude larger than this, as might be shown, for instance, by including nontoric bases.

All these models have in common that the Higgs and lepton doublets are localized on the same matter curve. As such, this curve must have nonzero genus to allow for the existence of vectorlike pairs [32]. Given the homology class of the doublet curve [11] and our restriction $\mathcal{S}_{7,9}=\overline{\mathcal{K}}$, the genus in question is indeed $g=1+9 / 2 \overline{\mathcal{K}}^{3}>0$, since $\overline{\mathcal{K}}^{3} \geq 2$ by (10). It would be very interesting, albeit extremely difficult with current methods, to study the precise complex structure dependence of the number of Higgs doublets and other charged vectorlike pairs in this ensemble.

Furthermore, since our models have no additional (possibly massive) Abelian gauge symmetries, all Yukawa couplings relevant for the standard model are automatically realized perturbatively, as can be shown by an explicit study of codimension-three singularities [15]. However, this in turn also implies that certain proton decay operators compatible with the standard model gauge group will, in general, be present [11]. We expect that, in some corners of the moduli space, which incidentally could also support high-scale supersymmetry breaking, these operators can be suppressed. Another avenue could be to instead focus on " $F$-theory standard models" that have additional [U(1) $[18,33]$ or $R$ parity [12] ] selection rules and estimate their numbers in the toric base landscape. We leave this for future work.

One interesting aspect of our ensemble is gauge coupling unification without a manifest grand unified theory (GUT) origin at the compactification scale. It can be easily read off geometrically from the divisors on $B_{3}$, which the 7-branes supporting the gauge symmetries in the type IIB picture wrap. Because of our restriction $\mathcal{S}_{7,9}=\overline{\mathcal{K}}$, both $\mathrm{SU}(3)$ and $\mathrm{SU}(2)$ gauge symmetries are realized on anticanonical divisors $\left\{s_{9}=0\right\}$ and $\left\{s_{3}=0\right\}$ with class $\overline{\mathcal{K}}$. (Note that because $\overline{\mathcal{K}}$ is not rigid, its deformation moduli give rise to nonchiral charged matter at the compactification scale. They have to be stabilized suitably at low energies.) Therefore, the gauge couplings are $g_{3,2}^{2}=2 / \operatorname{vol}(\overline{\mathcal{K}})$ [25,34]. [The factor of 2 arises because, in $F$ theory, the normalization dictated by geometry is one where the Cartan generators satisfy $\operatorname{tr}_{\text {fund }}\left(T_{i} T_{j}\right)=C_{i j}$ with $C$ as the Cartan matrix. On the other hand, the particle physics convention necessary to determine the coupling is $\operatorname{tr}_{\text {fund }}\left(T_{i} T_{j}\right)=\delta_{i j} / 2$.] The $\mathrm{U}(1)_{Y}$ coupling is the inverse volume of the so-called height-pairing divisor $b \subset B_{3}$ [35], which has been computed in [15] and reduces to $b=5 \overline{\mathcal{K}} / 6$ in our ensemble. Therefore, we have the standard MSSM gauge coupling unification

$$
g_{3}^{2}=g_{2}^{2}=\frac{5}{3} g_{Y}^{2}=\frac{2}{\operatorname{vol}(\overline{\mathcal{K}})},
$$

which for our models is achieved at the compactification scale. While this scale as well as the actual values of the couplings will depend on the details of moduli stabilization, the relationship (12) is independent of Kähler moduli. It would be interesting to see if this relationship originates from an honest geometric realization of a GUT structure. Given the known connection of our ensemble to a PatiSalam $\left[\mathrm{SU}(4) \times \mathrm{SU}(2)^{2}\right] / \mathbb{Z}_{2}$ model $[11,15]$, we expect an underlying $\mathrm{SO}(10)$.

Our results may provide phenomenological motivation for the study of new moduli stabilization scenarios. Specifically, though gauge coupling unification is automatic in our ensemble, it is natural to ask whether the correct value $\alpha_{\mathrm{GUT}} \simeq 0.03$ can be obtained in canonical moduli stabilization schemes. For instance, the KachruKallosh-Linde-Trivedi (KKLT) and large volume scenarios [36] assume that cycles are at sufficiently large volume to safely ignore string world sheet instanton corrections to the Kähler potential. This is essential because it is not known how to systematically compute and control all instanton contributions in $\mathcal{N}=1$ backgrounds. A necessary condition for safely ignoring these corrections is to have $\operatorname{vol}(C)>1$ (in string units) for all curves $C \subset B_{3}$. This condition defines a stretched out subset of the Kähler cone [37], where it was also shown that the Kähler cones become increasingly narrow for increasing $h^{1,1}$. In effect, this forces toric divisors to be increasingly large in order to safely ignore world sheet instantons, leading to smaller gauge couplings, because on toric $B_{3}$ the class $\overline{\mathcal{K}}$ is the sum of all toric divisors. Brief calculations suggest that the correct $\alpha_{\mathrm{GUT}}$ cannot be obtained in this controlled regime, in which case realistic models in our scenario are not consistent with the KKLT or large volume scenarios. Firmly concluding this requires a more detailed study, but we emphasize that it would not rule out our models and instead motivate the study of new moduli stabilization scenarios that allow for the observed value of gauge couplings.

Our compactifications also exhibit D3-branes. These sectors generically give rise to $\mathrm{U}(1)$ gauge theories that could be cosmologically relevant as dark photons. Each has 
its own open string moduli, the position of the D3-brane, which are massless at tree level but may be stabilized by nonperturbative effects due to their appearance in instanton prefactors [38]. However, since all but one of the toric divisors are rigid in the geometries we study, it is likely that there are many instanton corrections to the superpotential. Each instanton acts with an attractive force on the D3-brane, pulling it toward the associated divisor, but the existence of many such contributions would provide competing forces that stabilize the $D 3$-brane away from each toric divisor. In particular, due to these competing effects we see a priori no reason that the D3-branes should be stabilized anywhere near the SU(3) or SU(2) 7-branes, in which case jointly charged matter in the form of 3-7 strings decouple from the spectrum. Such a scenario gives rise to numerous dark photon sectors that have cosmological effects only through kinetic mixing with the visible sector and with one another. It would be interesting to study these sectors further, in light of current and future dark photon experiments.

We note that gravity cannot be decoupled in our ensemble since the standard model gauge divisors are in the anticanonical class, yielding a nontrivial interplay between gravity and the visible sector. This interplay arises due to the details of our construction and could lead to other interesting interactions between particle physics and cosmology. At the level of toric geometry, the models of our ensemble differ from one another by how the facets are triangulated. This does not affect the structure of the anticanonical divisors that realize $\mathrm{SU}(3)$ and $\mathrm{SU}(2)$, and thus the particle physics spectrum is insensitive to details of the triangulation; it is, after all, what gives rise to the large number of standard models in our construction. The triangulation is critical, however, for moduli stabilization. For instance, the classical Kähler potential on Kähler moduli is determined by triangulation-dependent topological intersections. This affects numerous aspects of the cosmology of these models, including inflation.

This visible sector universality in the midst of cosmological diversity might lead one to question the extent to which these should be counted as truly different models. Though a natural question, it has a clear answer: since the geometries are different, they lead to distinct four-dimensional effective theories below the Kaluza-Klein scale, each of which could give rise to numerous metastable vacua. Instead, our view is that the universal structure in the visible sector provides some evidence for a long-held hope in the string landscape, that, despite large numbers of vacua, there could exist semiuniversal features that lead to meaningful statistical predictions.

We thank Craig Lawrie and Cody Long for helpful discussions and Jonathan Carifio for providing the dataset of [14]. M. C. and L. L. are supported by DOE Award
No. DE-SC0013528. M. C. further acknowledges the support by the Fay R. and Eugene L. Langberg Endowed Chair and the Slovenian Research Agency. J. H. is supported by NSF Grant No. PHY-1620526.

[1] C. Vafa, Nucl. Phys. B469, 403 (1996).

[2] T. Weigand, Proc. Sci. TASI2017, 016 (2018); M. Cvetič and L. Lin, Proc. Sci. TASI2017, 020 (2018).

[3] S. Ashok and M. R. Douglas, J. High Energy Phys. 01 (2004) 060; W. Taylor and Y.-N. Wang, J. High Energy Phys. 12 (2015) 164; J. Halverson, C. Long, and B. Sung, Phys. Rev. D 96, 126006 (2017).

[4] R. Blumenhagen, F. Gmeiner, G. Honecker, D. Lüst, and T. Weigand, Nucl. Phys. B713, 83 (2005); F. Gmeiner, R. Blumenhagen, G. Honecker, D. Lüst, and T. Weigand, J. High Energy Phys. 01 (2006) 004.

[5] L. B. Anderson, J. Gray, A. Lukas, and E. Palti, J. High Energy Phys. 06 (2012) 113; L. B. Anderson, A. Constantin, J. Gray, A. Lukas, and E. Palti, J. High Energy Phys. 01 (2014) 047; A. Constantin, Y.-H. He, and A. Lukas, Phys. Lett. B 792, 258 (2019).

[6] M. Cvetič, G. Shiu, and A. M. Uranga, Nucl. Phys. B615, 3 (2001); Phys. Rev. Lett. 87, 201801 (2001).

[7] R. Blumenhagen, M. Cvetič, P. Langacker, and G. Shiu, Annu. Rev. Nucl. Part. Sci. 55, 71 (2005).

[8] V. Braun, Y.-H. He, B. A. Ovrut, and T. Pantev, Phys. Lett. B 618, 252 (2005); 05 (2006) 043.

[9] V. Bouchard and R. Donagi, Phys. Lett. B 633, 783 (2006); V. Bouchard, M. Cvetič, and R. Donagi, Nucl. Phys. B745, 62 (2006).

[10] L. B. Anderson, Y.-H. He, and A. Lukas, J. High Energy Phys. 07 (2007) 049; L. B. Anderson, J. Gray, Y.-H. He, and A. Lukas, J. High Energy Phys. 02 (2010) 054.

[11] M. Cvetič, D. Klevers, D. K. M. Peña, P.-K. Oehlmann, and J. Reuter, J. High Energy Phys. 08 (2015) 087.

[12] M. Cvetič, L. Lin, M. Liu, and P.-K. Oehlmann, J. High Energy Phys. 09 (2018) 089.

[13] J. Halverson and J. Tian, Phys. Rev. D 95, 026005 (2017); J. Carifio, J. Halverson, D. Krioukov, and B. D. Nelson, J. High Energy Phys. 09 (2017) 157.

[14] R. Altman, J. Carifio, J. Halverson, and B. D. Nelson, J. High Energy Phys. 03 (2019) 186.

[15] D. Klevers, D. K. Mayorga Peña, P.-K. Oehlmann, H. Piragua, and J. Reuter, J. High Energy Phys. 01 (2015) 142.

[16] M. Cvetič and L. Lin, J. High Energy Phys. 01 (2018) 157.

[17] L. Lin, C. Mayrhofer, O. Till, and T. Weigand, J. High Energy Phys. 01 (2016) 098.

[18] L. Lin and T. Weigand, Nucl. Phys. B913, 209 (2016).

[19] M. Cvetič, A. Grassi, D. Klevers, and H. Piragua, J. High Energy Phys. 04 (2014) 010.

[20] D. K. Mayorga Pena and R. Valandro, J. High Energy Phys. 03 (2018) 107.

[21] K. Dasgupta, G. Rajesh, and S. Sethi, J. High Energy Phys. 08 (1999) 023; E. Witten, J. Geom. Phys. 22, 1 (1997).

[22] A. Collinucci and R. Savelli, J. High Energy Phys. 02 (2012) 015.

[23] K. Intriligator, H. Jockers, P. Mayr, D. R. Morrison, and M. R. Plesser, Adv. Theor. Math. Phys. 17, 601 (2013). 
[24] S. Sethi, C. Vafa, and E. Witten, Nucl. Phys. B480, 213 (1996).

[25] T. W. Grimm, Nucl. Phys. B845, 48 (2011).

[26] T. W. Grimm, M. Kerstan, E. Palti, and T. Weigand, J. High Energy Phys. 12 (2011) 004.

[27] D. S. Park, J. High Energy Phys. 01 (2012) 093; D. R. Morrison and D. S. Park, J. High Energy Phys. 10 (2012) 128.

[28] R. Donagi and M. Wijnholt, Adv. Theor. Math. Phys. 15, 1237 (2011); A. P. Braun, A. Collinucci, and R. Valandro, Nucl. Phys. B856, 129 (2012); J. Marsano and S. SchäferNameki, J. High Energy Phys. 11 (2011) 098; S. Krause, C. Mayrhofer, and T. Weigand, Nucl. Phys. B858, 1 (2012); T. W. Grimm and H. Hayashi, J. High Energy Phys. 03 (2012) 027.

[29] See Supplemental Material at http://link.aps.org/ supplemental/10.1103/PhysRevLett.123.101601 for a more detailed flux analysis

[30] M. Kreuzer and H. Skarke, Adv. Theor. Math. Phys. 2, 853 (1998).
[31] W. Stein et al., Sage Mathematics Software Ver. 8.4, The Sage Development Team, 2018, http://www.sagemath.org.

[32] M. Bies, C. Mayrhofer, C. Pehle, and T. Weigand, arXiv:1402.5144; M. Bies, C. Mayrhofer, and T. Weigand, J. High Energy Phys. 11 (2017) 081.

[33] L. Lin and T. Weigand, Fortschr. Phys. 63, 55 (2015).

[34] F. Bonetti and T. W. Grimm, J. High Energy Phys. 05 (2012) 019.

[35] M. Cvetič, T. W. Grimm, and D. Klevers, J. High Energy Phys. 02 (2013) 101.

[36] S. Kachru, R. Kallosh, A. Linde, and S. P. Trivedi, Phys. Rev. D 68, 046005 (2003); V. Balasubramanian, P. Berglund, J. P. Conlon, and F. Quevedo, J. High Energy Phys. 03 (2005) 007.

[37] M. Demirtas, C. Long, L. McAllister, and M. Stillman, arXiv:1808.01282.

[38] O. J. Ganor, Nucl. Phys. B499, 55 (1997); D. Baumann, A. Dymarsky, I. R. Klebanov, J. M. Maldacena, L.P. McAllister, and A. Murugan, J. High Energy Phys. 11 (2006) 031. 